\title{
Spontaneous vortex formation and Majorana zero mode in iron based superconductor.
}

1. Observation of a robust zero-energy bound state in iron-based superconductor Fe(Te,Se)

Authors: J. X. Yin, Zheng Wu, J. H. Wang, Z. Y. Ye, Jing Gong, X. Y. Hou, Lei

Shan, Ang Li, X. J. Liang, X. X. Wu, Li Jian, C.S. Ting,Z. Q. Wang, J. P. Hu, P. H. Hor, H. Ding and S. H. Pan.

Nature Physics 11, 543 (2015)

2. Quantum anomalous vortex and Majorana zero mode without external magnetic field

Authors: Kun Jiang, Xi Dai and Ziqiang Wang

arXiv:1808.07072

3. Nature of the zero energy bound state at the surface of the topological superconductor $\mathrm{Fe}(\mathrm{Se}, \mathrm{Te})$

Authors: T. Machida, Y. Sun, S. Pyon, S. Takeda, Y. Kohsaka, T. Hanaguri, T. Sasagawa and T. Tamegai.

arXiv:1812.08995

Recommended with a Commentary by Patrick A. Lee, MIT

In a recent commentary in January, 2018, Fa Wang reported on what looked like a gift from Nature. The iron based superconductor $F e T e_{1-x} S e_{x}(x=0.45)$ has a band inversion which creates a topological Dirac surface state. The fact that this is also a superconductor with a rather large gap allows one to realize the Fu-Kane proposal [1] of creating a system that supports Majorana edge modes, without the need to proximity couple to another superconductor. It has long been suggested that the vortex core of this state supports a Majorana zero mode. Indeed scanning tunneling spectroscopy (STS) performed at $0.55 \mathrm{~K}$ revealed zero bias peaks within the resolution. [2] This is a promising and exciting development, even though many questions remain to be answered, such as what happens at lower temperature and better resolution and why only 20 percent of the vortices show this zero bias peak. These questions are addressed in one of our recommended papers, Machida et al. However, we first turn our attention to an interesting point already made by Fa Wang, namely, there was an earlier report of zero bias peak in the same system in zero magnetic field that were tied to Fe interstitial defects. [See recommended article by Yin et al.] At that time the topological nature of this material was not known, and the observation appeared to be quite mysterious. 
With the new knowledge, it is high time to revisit this issue to see if one can come up with an explanation. This is the subject of the recommended paper by Jiang et al.

Jiang et al made two separate claims. The first is that in a superconductor with strong spin-orbit coupling, an magnetic impurity that is exchange coupled to the total angular momentum $j$ of the electrons can can spontaneously generate a vortex at the impurity site, i.e. the ground state has a phase winding of the pair phase of $2 \pi$, provided the exchange coupling is sufficiently strong. Furthermore, this vortex has the feature that the usual CarolideGennes states inside the vortex core are largely cleared out, leaving only the $j=0$ states inside the gap. Secondly, if the superconductor happens to be a topological one, a relatively isolated Majorana bound state is formed at zero energy. The second observation follows quite naturally from the first which we discuss in a bit more detail below.

In a strong spin orbit coupled system, the electron states around a vortex is labeled by the total angular momentum which is the sum of the orbital and spin contributions. In the usual vortex core, there are many bound states first identified by Caroli and deGennes (CdG) with energies given by half-integer multiples of $\Delta^{2} / E_{F}$ where $\Delta$ is the superconducting gap energy. This energy spacing is quite small and is detrimental to the coherence of the Majorana zero mode because they are easily excited thermally. Jiang et al assumed an exchange coupling $J_{K}$ between the magnetic moment of the impurity and the total angular momentum of the electrons. A given ordering of the magnetic moment pushes the states that carry angular momentum to the continuum above the gap, leaving just the $j=0$ ones behind. Next, Jiang et al compared the energy of a vortex that is spontaneously formed with a state that is vortex free. In the vortex free configuration the pairing amplitude is suppressed near the impurity, but not all the way to zero, and the suppression extends over a larger distance compared with the vortex. The comparison of the energies requires a numerical solution of the self-consistent equation and they found that for the parameters appropriate to $F e T e_{1-x} S e_{x}(x=0.45)$, a vortex will spontaneously form for a reasonable value of $J_{K}$. These vortices appear as vortex anti-vortex pairs with oppositely aligned moments on the surface layer.

It should be noted that $F e T e_{1-x} S e_{x}$ is unusual in that the Fermi energy is extremely small, of order $10 \mathrm{meV}$ while the energy gap is quite large, of order $1.5 \mathrm{meV}$, so that the ratio of $\Delta / E_{F}$ is only about 0.15 . As a result the coherence length is extremely short. In a more standard superconductor, the exchange coupling will extend only to the scale of the inverse Fermi momentum, which is normally much less than the coherence length. In this case the exchange coupling is rather ineffective and it can be expected that a very large $J_{K}$ of the order of $E_{F}$ is needed to spontaneously create the vortex. Perhaps this is the reason why this phenomenon has not been reported before.

The separation between the $\mathrm{CdG}$ states is estimated to be $200 \mu \mathrm{eV}$ which is comparable to the $250 \mu \mathrm{eV}$ energy resolution of the the study in ref. [2]. Machida et al reported significant advances on the experimental front. The energy resolution of their STS has been improved to $20 \mu \mathrm{eV}$ and the temperature can be as low as $85 \mathrm{mK}$, compared with $0.5 \mathrm{~K}$ in the earlier work. Figure 1 shows the STS scans of the spectra taken near the core of two vortices at a small magnetic field of 1T. Figure 1c shows a clear zero bias peak within the resolution while figure $1 \mathrm{~d}$ shows its absence. With a coarser resolution, a broadened version of figure 1a and 1b may lead one to the opposite conclusion. By fitting all the peaks and making a histogram, they show that the probability of finding a peak within $20 \mu \mathrm{eV}$ of zero energy is much larger than at higher energies. This is strong evidence that the zero bias peak is special 
and is a strong candidate for the predicted Majorana zero mode. Interestingly, they found that the probability of finding this zero bias peak drops smoothly from $80 \%$ to about $10 \%$ when the magnetic field is increased from $1 \mathrm{~T}$ to $6 \mathrm{~T}$. There appears to be coupling between vortices even when they are far apart compared with the very short coherence length. This helps explains the variability in the observations of these zero bias peaks in earlier reports but the mechanism of this coupling remains unknown.

In this study Machida et al focused on samples with a small amount of Fe defects. In samples with higher Fe defect concentration, they find zero bias peaks located at some of the defects and not others, in broad agreement with the earlier report by Yin et al. ( $\mathrm{T}$. Hanaguri, private communications.) It will be of great interest to study these peaks with the improved resolution, which hopefully will be on their agenda.
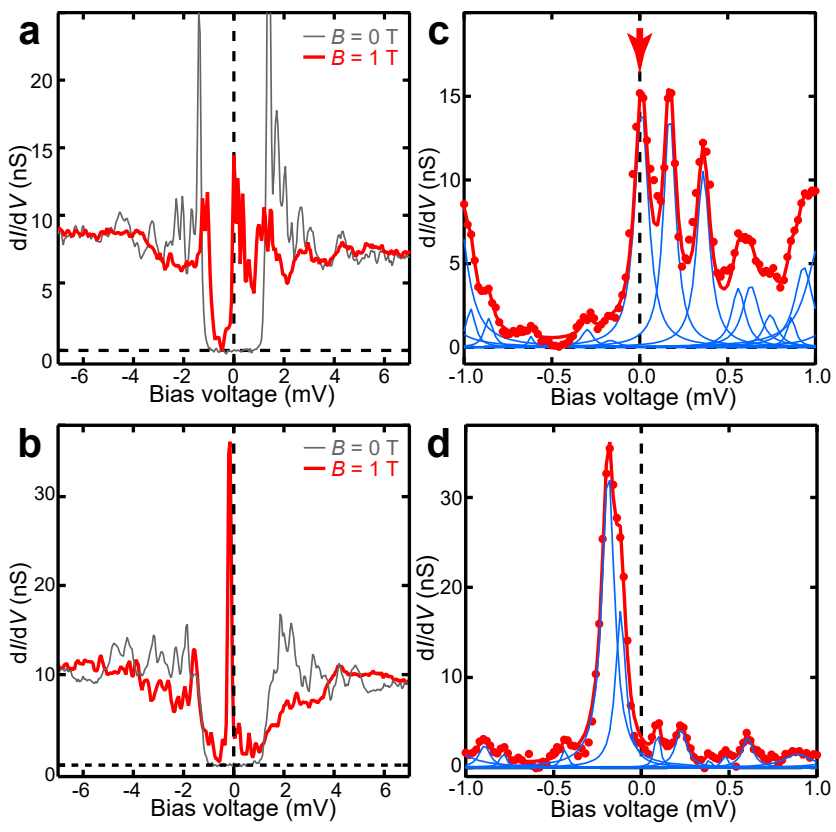

Figure 1: Top and bottom panels are the STS scans near the core of two different vortices at $\mathrm{B}=1 \mathrm{~T}$. The energy resolution is $20 \mu \mathrm{eV}$. The right panels are the expanded view of the left. Arrow in the top right panel indicates a zero bias peak. From Machida et al.

\section{References}

[1] L. Fu and C. L. Kane, Phys. Rev. Lett. 100, 096407 (2008).

[2] Dongfei Wang et al, Science 362, 333 (2018). 